\title{
ON THE DUNFORD-PETTIS PROPERTY
}

\author{
J. BOURGAIN
}

\begin{abstract}
It is shown that the Banach spaces $C_{L^{1}}$ and $L_{C}^{1}$ and their duals have the Dunford-Pettis property. Some other more local problems are also solved.
\end{abstract}

1. Introduction. We say that a Banach space $X$ has the Dunford-Pettis (D-P) property provided for all weakly null sequences $\left(x_{n}\right)$ in $X$ and $\left(x_{n}^{*}\right)$ in $X^{*}$, we have that $\lim x_{n}^{*}\left(x_{n}\right)=0$.

We call a subset $A$ of $X$ weakly conditionally compact (WCC), if any sequence in $A$ has a weak Cauchy subsequence. It was shown by $\mathrm{H}$. Rosenthal [9] that $A$ is WCC if and only if $A$ is bounded and does not contain a sequence which is equivalent to the usual $l^{1}$-basis. It is easily seen that the D-P property means that weakly null sequences in $X$ are uniformly convergent on WCC subsets of $X^{*}$ and vice versa.

A. Grothendieck proved that spaces of continuous functions (hence also $l^{\infty}$ ) and Lebesgue spaces $L^{1}(\mu)$ are D-P spaces. More generally, $\mathfrak{L}^{\infty}$ and $\mathcal{L}^{1}$-spaces (see [7] for definitions) have the D-P property.

We will show that $C_{L^{1}}$ and $L_{C}^{1}$-spaces are also D-P, which was an open question. As will be clear from what follows, the argument here is more involving and uses a "nonlinear" technique. Our results also allow us to show that $l^{2}$ is not finitely complemented in $C_{L^{1}}$, a problem raised in [3].

We use the notation $\left(\varepsilon_{i}\right)$ for the sequence of Rademacher functions on $[0,1]$. Let us recall the following elementary property.

LEMMA 1. If $\left(x_{i}\right)$ is a finite sequence in a Banach space $X$, then $\int\left\|\Sigma_{i} \varepsilon_{i}(\omega) x_{i}\right\| d \omega>$ $\max _{i}\left\|x_{i}\right\|$.

The next result is a particular case of a more general theorem (see [1] and [11]) and is in fact easily derived from the Rosenthal characterization theorem [1].

LEMMA 2. If $X$ is a Banach space and $\left(x_{i}\right)_{i-1,2, \ldots}$ a WCC sequence in $X$, then the sequence $\left(x_{i} \otimes \varepsilon_{i}\right)_{i=1,2, \ldots}$ is WCC in $L_{X}^{1}$.

The only interest of Lemma 2 here is the simplification of some arguments.

Received by the editors September 5, 1979.

1980 Mathematics Subject Classification. Primary 46E40, 46B99, 28 B99.

Key words and phrases. Dunford-Pettis property. 
2. The basic result. Let $X$ be a Banach space. Denote $\bigoplus_{\infty} X$ the $l^{\infty}$-sum of spaces $X$. If $\xi \in \bigoplus_{\infty} X$, take $|\xi|=\left(\left\|\xi_{1}\right\|,\left\|\xi_{2}\right\|, \ldots\right)$ which is an element of $l^{\infty}$. For $\eta \in\left[\bigoplus_{\infty} X\right]^{*}$, we let $|\eta|$ be the positive element of $\left(l^{\infty}\right)^{*}$, given by $\langle\alpha,|\eta|\rangle=$ $\sup \left\{\langle\xi, \eta\rangle ; \xi \in \bigoplus_{\infty} X\right.$ and $\left.|\xi|<\alpha\right\}$ for $\alpha \in l_{+}^{\infty}$. It is indeed clear that $|\eta|$ is linear and $\||\eta|\|=\|\eta\|$.

This section is devoted to the proof of our main result, namely

THEOREM 1. The space $\bigoplus_{\infty} L^{1}$ has the $D-P$ property.

The proof requires additional lemmas.

Using the same notation as presented at the beginning of the section, we show

Lemma 3. If $A$ is a WCC subset of $\left[\bigoplus_{\infty} X\right]^{*}$, then $\{|\eta| ; \eta \in A\}$ is equicontinuous.

Proof. Suppose $\{|\eta| ; \eta \in A\}$ is not equicontinuous. Then there exist $\varepsilon>0$, a sequence $\left(\eta_{i}\right)$ in $A$ and a sequence $\left(M_{i}\right)$ of subsets of $N$, such that $\left|\eta_{i}\right|\left(M_{i}\right)>\varepsilon$ and $\left|\eta_{i}\right|\left(M_{j}\right)<3^{-j} \varepsilon$ for $j>i$. We will obtain a contradiction on the WCC of $A$ by showing that $\left(\eta_{i} \otimes \varepsilon_{i}\right)$ is an $l^{1}$-basis. So fix an integer $k$ and choose scalars $a_{1}, \ldots, a_{k}$. Define $N_{i}=M_{i} \backslash \cup{ }_{j=i+1}^{k} M_{j}$ for $i=1, \ldots, k$. Clearly

$$
\begin{aligned}
\left|\eta_{i}\right|\left(N_{i}\right) & >\left|\eta_{i}\right|\left(M_{i}\right)-\sum_{j=i+1}^{k}\left|\eta_{i}\right|\left(M_{j}\right) \\
& >\varepsilon-\sum_{j=i}^{k} 3^{-j} \varepsilon>\frac{\varepsilon}{2}
\end{aligned}
$$

Now, applying Lemma 1

$$
\begin{aligned}
\int\left\|\sum_{i=1}^{k} a_{i} \varepsilon_{i}(\omega) \eta_{i}\right\| d \omega & >\sum_{j=1}^{k} \int\left|\sum_{i=1}^{k} a_{i} \varepsilon_{i}(\omega) \eta_{i}\right|\left(N_{j}\right) d \omega \\
& >\sum_{j=1}^{k}\left|a_{j}\right|\left|\eta_{j}\right|\left(N_{j}\right)>\frac{\varepsilon}{2} \sum_{j=1}^{k}\left|a_{j}\right|,
\end{aligned}
$$

proving the lemma.

The Banach space $X$ will be $L^{1}(\mu)$, where $\mu$ is some probability measure.

LeMma 4. Let $A$ be a WCC subset of $\bigoplus_{\infty} L^{1}, \nu$ a positive element of $\left(l^{\infty}\right)^{*}$ and $\varepsilon>0$. Then there exist some $\zeta$ in $\bigoplus_{\infty} L_{+}^{1},\|\zeta\|=1$ and some $\delta>0$ such that $\nu(M)<\varepsilon$ for each $\xi \in A$, where $M=\left\{n \in N ; \int_{S} \zeta_{n} d \mu<\delta\right.$ and $\int_{S} \xi_{n} d \mu>\varepsilon$ for some $S$ \}.

Proof. The requirement $\|\zeta\|=1$ is of course unimportant. Assume the claim untrue. Proceeding by induction, it is possible to obtain a sequence $\left(\xi^{i}\right)$ in $A$ such that $\nu\left(M_{i}\right)>\varepsilon$ for each $i$, where

$$
M_{i}=\left\{n \in \mathbf{N} ; \int_{S} \zeta_{n}^{i} d \mu<3^{-i} \varepsilon \text { and } \int_{S}\left|\xi_{n}^{i}\right| d \mu>\varepsilon \text { for some } S\right\}
$$

and $\zeta^{i} \in \bigoplus_{\infty} L^{1}$ is given by $\zeta_{n}^{i}=\max \left(\left|\xi_{n}^{j}\right| ; 1<j<i-1\right)$. The construction is again completely straightforward, so we omit details. 
There is an infinite subset $I$ of $\mathbf{N}$ such that $\left(M_{i}\right)_{i \in I}$ has the finite intersection property. We will show that $\left(\xi^{i} \otimes \varepsilon_{i}\right)_{i \in I}$ is equivalent to the $l^{1}$-basis, which will provide the required contradiction.

Fix $i_{1}<i_{2}<\cdots<i_{r}$ in $I$ and scalars $a_{1}, a_{2}, \ldots, a_{r}$. Take $n \in \cap_{s=1}^{r} M_{i_{s}}$. For each $s=1, \ldots, r$ there exist a set $S_{s}$ satisfying $\int_{S_{s}}\left|\xi_{n}^{i_{i}}\right| d \mu>\varepsilon$ and $\int_{S_{s}}\left|\xi_{n}^{i}\right| d \mu<3^{-s} \varepsilon$ for $i<i_{s}$. So if $T_{s}=S_{s} \backslash \cup_{t=s+1}^{r} S_{t}$, we obtain

$$
\begin{aligned}
\int_{T_{s}}\left|\xi_{n}^{i_{j}}\right| d \mu & >\int_{S_{s}}\left|\xi_{n}^{i_{j}}\right| d \mu-\sum_{t=s+1}^{r} \int_{S_{t}}\left|\xi_{n}^{i_{j}}\right| d \mu \\
& >\varepsilon-\sum_{t=s+1}^{r} 3^{-t} \varepsilon>\frac{\varepsilon}{2} .
\end{aligned}
$$

Finally, again by Lemma 1

$$
\begin{aligned}
\int\left\|\sum_{s} a_{s} \varepsilon_{s}(\omega) \xi^{i j}\right\| d \omega & >\int\left\|\sum_{s} a_{s} \varepsilon_{s}(\omega) \xi_{n}^{i}\right\| d \omega \\
& >\sum_{t=1}^{r} \iint_{T_{t}}\left|\sum_{s} a_{s} \varepsilon_{s}(\omega) \xi_{n}^{i_{s}}\right| d \mu d \omega \\
& >\sum_{t=1}^{r}\left|a_{t}\right| \int_{T_{t}}\left|\xi_{n}^{i}\right| d \mu>\frac{\varepsilon}{2} \sum_{t=1}^{r}\left|a_{t}\right| .
\end{aligned}
$$

This completes the proof.

We assume $\left(\xi^{i}\right)$ a weakly null sequence in $\bigoplus_{\infty} L^{1},\left(\eta_{i}\right)$ a weakly null sequence in $\left(\bigoplus_{\infty} L^{1}\right)^{*}$ such that $\left\|\xi^{i}\right\|=\left\|\eta_{i}\right\|=1$ and $\left\langle\xi^{i}, \eta_{i}\right\rangle>\rho(\rho>0)$ and will work towards a contradiction.

If $\mathcal{E}$ is a sub- $\sigma$-algebra, $\mu^{\prime}<<\mu$ and $f \in L^{1}\left(\mu^{\prime}\right)$, then $E\left[f, \mathcal{E}, \mu^{\prime}\right]$ will denote the conditional expectation of $f$ with respect to $\delta$ in the space $L^{1}\left(\mu^{\prime}\right)$.

LEMMA 5. There exist $\theta$ in $\bigoplus_{\infty} L_{+}^{1}$, a sequence $\left(f^{j}\right)$ in $\bigoplus_{\infty} L^{\infty}$ and a sequence $\left(\lambda_{j}\right)$ in $\left(\bigoplus_{\infty} L^{1}\right)^{*}$ such that the following conditions are fulfilled.

(1) $\left\|f^{j}\right\|=\sup _{n}\left\|f_{n}^{j}\right\|_{\infty} \leqslant 1$.

(2) For each $n$, the sequence $\left(f_{n}^{j}\right)_{j}$ is a martingale difference sequence in the space $L^{1}\left(\mu_{n}\right)$, where $d \mu_{n}=\theta_{n} d \mu$.

(3) $\left\langle\Delta^{j}, \lambda_{j}\right\rangle>\rho / 4$, where $\Delta^{j} \in \bigoplus_{\infty} L^{1}$ is defined by $\Delta_{n}^{j}=f_{n}^{j} \theta_{n}$.

(4) $\left(\lambda_{j}\right)$ is a subsequence of $\left(\eta_{i}\right)$.

Proof. Let $\nu_{i}=\left|\eta_{i}\right|$ and $\nu=\Sigma_{i} 2^{-i} \nu_{i}$. Take $\iota=\rho / 12$. By Lemma 3, there is some $0<\varepsilon<\iota$ so that $\sup _{i} \nu_{i}(M)<\iota$ if $M \subset \mathbf{N}$ and $\nu(M)<\varepsilon$. Let then $\zeta$ and $\delta$ be as in Lemma 4, applied with $A=\left\{\xi^{i} ; i\right\}$.

Take $\theta=\delta^{-1} \zeta$. For each $n$ and $i$, let $S_{n}^{i}=\left[\left|\xi_{n}^{i}\right|>\theta_{n}\right]$ and $\psi_{n}^{i}=\left(1-\chi_{n}^{i}\right) \xi_{n}^{i}$, where $\chi_{n}^{i}$ is the characteristic function of $S_{n}^{i}$. Let also $M_{i}=\left\{n ;\left\|\xi_{n}^{i}-\psi_{n}^{i}\right\|_{1}>\varepsilon\right\}$ for each $i$. Clearly

$$
\int_{S_{n}^{i}} \zeta_{n} d \mu<\delta\left\|\xi_{n}^{i}\right\|_{1}<\delta \text { and }\left\|\xi_{n}^{i}-\psi_{n}^{i}\right\|_{1}=\int_{S_{n}^{i}}\left|\xi_{n}^{i}\right| d \mu .
$$

So $\nu\left(M_{i}\right)<\varepsilon$, by the choice of $\zeta$ and $\delta$.

Since $\psi_{n}^{i}<\theta_{n}$, we may write $\psi_{n}^{i}=g_{n}^{i} \theta_{n}$ for some $g_{n}^{i} \in L^{\infty}(\mu) ;\left\|g_{n}^{i}\right\|_{\infty}<1$. We construct the $f^{j}$ and $\lambda_{j}$ inductively. 
Suppose $f^{j}$ obtained such that each $f_{n}^{j}$ is $\varepsilon_{n}^{j}$-measurable for some finite algebra $\xi_{n}^{j}$, where the number $d=d_{j}$ of atoms of $\xi_{n}^{j}$ does not depend on $n$. Denote $I_{n, e}$ $(1<e<d)$ the atoms of $\mathcal{E}_{n}^{j}$ and consider for each $e=1, \ldots, d$ the weakly null sequence $\left(\alpha^{i}(e)\right)_{i}$ in $l^{\infty}$, defined by $\alpha_{n}^{i}(e)=\int_{I_{n,}} \xi_{n}^{i} d \mu$.

Let $B$ be the direct sum of $d$ copies of the space $l^{\infty}$. We may introduce the operator $\Gamma: B \rightarrow \bigoplus_{\infty} L^{1}(\mu)$, defined by

$$
\Gamma_{n}(\beta(1), \ldots, \beta(d))=\sum_{e=1}^{d} \frac{\beta_{n}(e)}{\mu_{n}\left(I_{n, e}\right)} \pi_{n, e} \theta_{n},
$$

where $\pi_{n, e}$ is the characteristic function of $I_{n, e}$. It is indeed clear that $\left\|\Gamma_{n}(\beta(1), \ldots, \beta(d))\right\|_{1}<\Sigma_{e}\left|\beta_{n}(e)\right|<\Sigma_{e}\|\beta(e)\|$ and hence $\Gamma$ is bounded.

The sequence $\left(\Gamma^{*}\left(\eta_{i}\right)\right)$ is weakly null in $B^{*}$. Since $l^{\infty}$ is a D-P space, $B$ is also D-P and therefore $\left\langle\alpha^{i}(1), \ldots, \alpha^{i}(d), \Gamma^{*}\left(\eta_{i}\right)\right\rangle$ tends to 0 for $i \rightarrow \infty$.

Fix some $i$ large enough to ensure that $\left\langle\sigma^{i}, \eta_{i}\right\rangle \mid<\varepsilon$, where $\sigma^{i}=$ $\Gamma\left(\alpha^{i}(1), \ldots, \alpha^{i}(d)\right)$. Remark that $\left\|\sigma_{n}^{i}\right\|<\Sigma_{e}\left|\alpha_{n}^{i}(e)\right|<\left\|\xi_{n}^{i}\right\|$ and thus $\left\|\sigma^{i}\right\|<1$.

A standard argument allows us to construct finite algebra's $\left(\mathcal{E}_{n}^{j+1}\right)$ such that $\mathcal{E}_{n}^{j} \subset \mathcal{E}_{n}^{j+1},\left\|g_{n}^{i}-E\left[g_{n}^{i}, \mathcal{E}_{n}^{j+1}, \mu_{n}\right]\right\|_{\infty}<\varepsilon /\left\|\mu_{n}\right\|$ and the number of atoms of $\mathcal{E}_{n}^{j+1}$ is bounded.

Define $f^{j+1}$ by $2 f_{n}^{j+1}=E\left[g_{n}^{i}, \varepsilon_{n}^{j+1}, \mu_{n}\right]-E\left[g_{n}^{i}, \varepsilon_{n}^{j}, \mu_{n}\right]$ if $n \in N_{i}=\mathbf{N} \backslash M_{i}$ and $f_{n}^{j+1}=0$ if $n \in M_{i}$. Take $\lambda_{j+1}=\eta_{i}$. It remains to verify (3). For each $n$,

$$
\sigma_{n}^{i}-E\left[g_{n}^{i}, \varepsilon_{n}^{j}, \mu_{n}\right] \theta_{n}=\sum_{e} \frac{\int\left(\xi_{n}^{i}-g_{n}^{i} \theta_{n}\right) \pi_{n, e} d \mu}{\mu_{n}\left(I_{n, e}\right)} \pi_{n, e} \theta_{n}
$$

and therefore $\left\|\sigma_{n}^{i}-E\left[g_{n}^{i}, \xi_{n}^{j}, \mu_{n}\right] \theta_{n}\right\|_{1} \leqslant\left\|\xi_{n}^{i}-\psi_{n}^{i}\right\|_{1}$. So

$$
\begin{aligned}
\left\langle 2 \Delta^{j+1}, \lambda_{j+1}\right\rangle= & \left\langle\left(2 f_{n}^{j+1} \theta_{n}\right)_{n \in N_{i}}, \eta_{i}\right\rangle \geqslant\left\langle\left(g_{n}^{i} \theta_{n}\right)_{n \in N_{i}}, \eta_{i}\right\rangle-\left\langle\left(\sigma_{n}^{i}\right)_{n \in N_{i}}, \eta_{i}\right\rangle \\
& -\sup _{n \in N_{i}}\left\|g_{n}^{i} \theta_{n}-E\left[g_{n}^{i}, \varepsilon_{n}^{j+1}, \mu_{n}\right] \theta_{n}\right\|_{1}-\sup _{n \in N_{i}}\left\|\sigma_{n}^{i}-E\left[g_{n}^{i}, \varepsilon_{n}^{j}, \mu_{n}\right] \theta_{n}\right\|_{1} \\
\geqslant & \left\langle\left(\psi_{n}^{i}\right)_{n \in N_{i}}, \eta_{i}\right\rangle-\left\langle\left(\sigma_{n}^{i}\right)_{n \in N_{i}}, \eta_{i}\right\rangle-2 \varepsilon \\
\geqslant & \left\langle\xi^{i}, \eta_{i}\right\rangle-\left\langle\sigma^{i}, \eta_{i}\right\rangle-\left\langle\left(\xi_{n}^{i}\right)_{n \in M_{i}}, \eta_{i}\right\rangle-\left\langle\left(\sigma_{n}^{i}\right)_{n \in M_{i}}, \eta_{i}\right\rangle-3 \varepsilon \\
\geqslant & \rho-\left(\left\|\xi^{i}\right\|+\left\|\sigma^{i}\right\|\right) \nu_{i}\left(M_{i}\right)-4 \varepsilon>\rho-2 \imath-4 \varepsilon
\end{aligned}
$$

or $\left\langle\Delta^{j+1}, \lambda_{j+1}\right\rangle \geqslant \rho / 4$. This proves the lemma.

Starting from Lemma 5, we end the proof of Theorem 1.

Proof of Theorem 1. Take $0<\kappa<\rho / 4\|\theta\|$. Since $\left(\lambda_{j}\right)$ is weakly null, it is possible to find a finitely supported sequence $\left(a_{j}\right)$ of positive scalars such that $\sum a_{j}=1$ and $\left\|\sum_{j} a_{j} \varepsilon_{j} \lambda_{j}\right\| \leqslant \kappa$ for all signs $\varepsilon_{j}= \pm 1$. Consequently

$$
\int\left\|\sum_{j} a_{j} \varepsilon_{j}(\omega) \lambda_{j}\right\| d \omega<\kappa .
$$

Take now for each $\omega$ the (Riesz) product

$$
R_{n}(\omega)=\prod_{j}\left(1+\varepsilon_{j}(\omega) f_{n}^{j}\right) \theta_{n}
$$


Clearly $R_{n}(\omega)$ is a positive function and $\left\|R_{n}(\omega)\right\|_{1}=\int R_{n}(\omega) d \mu=\left\|\theta_{n}\right\|_{1}$, using the fact that

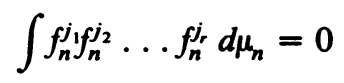

whenever $j_{1}<j_{2}<\cdots<j_{r}$.

So $R(\omega)=\left(R_{1}(\omega), R_{2}(\omega), \ldots\right)$ is a member of $\bigoplus_{\infty} L^{1}$ and $\|R(\omega)\|=\|\theta\|$. Therefore

$$
\begin{aligned}
\frac{\rho}{4} & >\int\left\|\sum_{j} a_{j} \varepsilon_{j}(\omega) \lambda_{j}\right\|\|R(\omega)\| d \omega \\
& \geqslant \int\left\langle R(\omega), \sum_{j} a_{j} \varepsilon_{j}(\omega) \lambda_{j}\right\rangle d \omega \\
& =\sum_{j} a_{j}\left\langle\int \varepsilon_{j}(\omega) R(\omega) d \omega, \lambda_{j}\right\rangle .
\end{aligned}
$$

But $\int \varepsilon_{j}(\omega) R_{n}(\omega) d \omega=f_{n}^{j} \theta_{n}=\Delta_{n}^{j}$ and thus $\rho / 4>\Sigma_{j} a_{j}\left\langle\Delta^{j}, \lambda_{j}\right\rangle$, a contradiction.

3. Consequences of the main result. The following observation will allow us to prove in certain cases the D-P property of a Banach space using the local structure of the space.

Proposition 2. Let $X$ be a Banach space and assume $X=\overline{\cup_{n} X_{n}}$ where $\left(X_{n}\right)$ is an increasing sequence of subspaces of $X$. If now $\bigoplus_{\infty} X_{n}$ is a D-P space, then $X$ also has D-P property.

Proof. Assume $\left(x_{i}\right)$ a weakly null sequence in $X$ and $\left(x_{i}^{*}\right)$ a weakly null sequence in $X^{*}$ such that $\left\langle x_{i}, x_{i}^{*}\right\rangle$ does not tend to null. It is clear that we may assume the $x_{i}$ in $\cup_{n} X_{n}$. Since the $X_{n}$ are increasing, it is possible to find a subsequence $\left(Y_{n}\right)$ of $\left(X_{n}\right)$, so that $x_{1}, \ldots, x_{n}$ belongs to $Y_{n}$, for each $n$. We will show that $\bigoplus_{\infty} Y_{n}$ fails the D-P property. Because $\bigoplus_{\infty} Y_{n}$ is complemented in $\bigoplus_{\infty} X_{n}$, also $\bigoplus_{\infty} X_{n}$ is not D-P, which will complete the proof. Denote $i_{n}: Y_{n} \rightarrow X$ the injection and $p_{n}$ : $\bigoplus_{\infty} Y_{n} \rightarrow Y_{n}$ the projection. Consider the sequence $\left(\xi^{i}\right)$ in $\bigoplus_{\infty} Y_{n}$, where the vector $\xi^{i}$ is defined by

$$
\begin{array}{ll}
\xi_{n}^{i}=0 & \text { if } n<i, \\
\xi_{n}^{i}=x_{i} & \text { if } n>i .
\end{array}
$$

Let $\mathcal{U}$ be a free ultrafilter on $\mathbf{N}$. Introduce the sequence $\left(\eta_{i}\right)$ in $\left[\bigoplus_{\infty} Y_{n}\right]^{*}$ by taking $\eta_{i}=\lim _{\mathcal{Q}} p_{n}^{*} i_{n}^{*}\left(x_{i}^{*}\right)$. Thus $\left\langle\xi^{i}, \eta_{i}\right\rangle=\lim _{\mathcal{Q}}\left\langle\xi_{n}^{i}, x_{i}^{*}\right\rangle=\left\langle x_{i}, x_{i}^{*}\right\rangle$.

It remains to verify that $\left(\xi^{i}\right)$ and $\left(\eta_{i}\right)$ are weakly null. So fix an infinite subset $M$ of $\mathbf{N}$ and $\delta>0$. Because $\left(x_{i}\right)$ is weakly null, there is a finitely supported sequence $\left(\lambda_{i}\right)_{i \in M}$ of positive scalars such that $\Sigma_{i} \lambda_{i}=1$ and $\left\|\Sigma_{i} \lambda_{i} \varepsilon_{i} x_{i}\right\|<\delta$ for all signs $\varepsilon_{i}= \pm 1$. Hence

$$
\begin{aligned}
\left\|\sum_{i} \lambda_{i} \xi^{i}\right\| & =\sup _{n}\left\|\sum_{i<n} \lambda_{i} x_{i}\right\| \\
& =\sup _{n}\left\|\frac{1}{2}\left(\sum_{i<n} \lambda_{i} x_{i}+\sum_{i>n} \lambda_{i} x_{i}\right)+\frac{1}{2}\left(\sum_{i<n} \lambda_{i} x_{i}-\sum_{i>n} \lambda_{i} x_{i}\right)\right\|
\end{aligned}
$$


is also bounded by $\delta$. Since $\left(x_{i}^{*}\right)$ is weakly null, there is a convex combination $\Sigma_{i \in M} \lambda_{i} x_{i}^{*}$ so that $\left\|\Sigma_{i} \lambda_{i} x_{i}^{*}\right\| \leqslant \delta$. Consequently $\left\|\Sigma_{i} \lambda_{i} p_{n}^{*} i_{n}^{*}\left(x_{i}^{*}\right)\right\|<\delta$ for each $n$ and hence also $\left\|\Sigma_{i} \lambda_{i} \eta_{i}\right\|<\delta$ as required.

Remark. Proposition 2 has no converse. Consider for instance the space $X=$ $\bigoplus_{1} l^{2}(n)$, thus the $l^{1}$-sum of the $l^{2}(n)$-spaces. Then $X$ has the Schur property and hence the D-P property. However, if $\left(X_{n}\right)$ is an increasing sequence of subspaces of $X$ so that $X=\bar{\cup}_{n} X_{n}$, then $\bigoplus_{\infty} X_{n}$ is never D-P. Indeed, since $X_{n}$ contain uniformly complemented Hilbert spaces of arbitrarily large dimension, $\bigoplus_{\infty} l^{2}(n)$ is a complemented subspace of $\bigoplus_{\infty} X_{n}$ and $\bigoplus_{\infty} l^{2}(n)$ fails the D-P property by Proposition 2 (in fact, $l^{2}$ is a complemented subspace of $\bigoplus_{\infty} l^{2}(n)$ ).

If $X$ and $Y$ are two Banach spaces, then

$$
d(X, Y)=\inf \left\{\|T\|\left\|T^{-1}\right\| ; T: X \rightarrow Y \text { is an isomorphism }\right\}
$$

is called the Banach-Mazur distance of $X$ and $Y$. We also recall the definition of an ultra-product of Banach spaces.

Definition. Let $I$ be a set, $\mathcal{Q}$ a free ultra-filter on $I$ and $\left(X_{i}\right)_{i \in I}$ a family of Banach spaces. Then we let $\left(X_{i}\right)_{Q}$ be the quotient of the space $\bigoplus_{\infty} X_{i}$ by its subspace $N_{\mathscr{Q}}=\left\{\left(x_{i}\right) ; \lim _{थ}\left\|x_{i}\right\|=0\right\}$.

For more details and general properties about ultra-products, we refer the reader to [4], [5], [7] and [13]. The following result is due to J. Stern.

Proposition 3. If $X$ is a Banach space, then $X^{* *}$ (and consequently any even dual of $X$ ) is isometric to a 1-complemented subspace of some ultra-product $X_{\text {Q }}$ of $X$.

The next result about the local structure of ultraproducts is straightforward from the definition (cf. [7, p. 119]).

Proposition 4. Let $X$ be a Banach space, $m$ a positive integer, $E$ a finite dimensional Banach space and $\lambda<\infty$. Assume that for any subspace $U$ of $X$, $\operatorname{dim} U=m$, there exists a subspace $V$ of $X$ such that $U \subset V$ and $d(V, E)<\lambda$. Then the same holds for any ultra-product $X_{\mathcal{Q}}$ of $X$.

For any positive integer $p$, denote $E_{p}$ the $l^{\infty}$-sum of $p$ copies of the space $l^{1}(p)$.

LEMMA 6. For any integer $m$ and $\varepsilon>0$, there exists an integer $p=p(m, \varepsilon)$ such that if $U$ is an $m$-dimensional subspace of some $E_{q}$ space, then there exists a subspace $V$ of $E_{q}$ satisfying $U \subset V$ and $d\left(V, E_{p}\right)<1+\varepsilon$.

This phenomenon is the same as for $l^{1}$ and $l^{\infty}$ spaces. The proof is also completely similar (cf. [7, p. 197]).

THeOReM 5. Let $X$ be a Banach space and $\lambda<\infty$ with the following property.

For any finite dimensional subspace $U$ of $X$, there exists a subspace $V$ of $X$ such that $U \subset V$ and $d\left(V, E_{p}\right)<\lambda$, for some $p$.

Then

(1) any ultra-product $X_{\mathcal{Q}}$ of $X$ is D-P,

(2) all duals of $X$ are $D-P$. 
Proof. Using Proposition 3, the second assertion is clearly a consequence of the first. As a consequence of Lemma 6, we see that in fact the following condition is satisfied.

For any integer $m$, there exists an integer $p=p(m)$ such that if $U$ is a subspace of $X, \operatorname{dim} U=m$, then there is a subspace $V$ of $X$ satisfying $U \subset V$ and $d\left(V, E_{p}\right)<\lambda^{\prime}\left(\lambda^{\prime}>\lambda\right)$.

Now, by Proposition 4, any ultra-product $X_{\mathscr{Q}}$ has the same property. Therefore, any separable $Y$ of $X_{\mathscr{Q}}$ is contained in a subspace $Z$ of $X_{\mathscr{Q}}$ of the form $Z={\overline{U_{n} V_{n}}}_{\text {, }}$ where $\left(V_{n}\right)$ is an increasing sequence of spaces for which $d\left(V_{n}, E_{p_{n}}\right)<\lambda^{\prime}$, for some sequence $\left(p_{n}\right)$ of integers.

In order to show that $X_{\mathscr{Q}}$ is D-P, it is sufficient to prove that each such space $Z$ has D-P property. By Proposition, it is enough to show that $\bigoplus_{\infty} V_{n}$ is D-P. But $\bigoplus_{\infty} V_{n}$ is isomorphic to $\bigoplus_{\infty} E_{p_{n}}$, which is a complemented subspace of $\bigoplus_{\infty} l^{1}$ and hence of $\bigoplus_{\infty} L^{1}$. So Theorem 1 completes the proof.

Let us now consider the space $C_{L^{1}}$ of continuous $L^{1}$-valued functions and the space $L_{C}^{1}$ of Bochner integrable functions with values in $C$. For details about $L_{C}^{1}$ and its dual $\left(L_{C}^{1}\right)^{*}$, we refer the reader to [2].

As is well known, $C(K)$-spaces are $\mathfrak{L}_{1+}^{\infty}$-spaces and $L^{1}(\mu)$-spaces are $\mathfrak{L}_{1+}^{1}$-spaces (cf. [7, pp. 197-199]). The next result is an analogue for $L_{C}^{1}$ and $C_{L^{1}}$-spaces and is obtained using the same techniques.

Proposition 6. The spaces $C_{L^{1}}$ and $\left(L_{C}^{1}\right)^{*}$ have $E_{p}$-local structure. Or more precisely, if $U$ is a finite dimensional subspace of one of these spaces and $\varepsilon>0$, there exists a finite dimensional subspace $V$ satisfying $U \subset V$ and $d\left(V, E_{p}\right)<1+\varepsilon$ for some integer $p$.

Thus applying Theorem 5 , we find

Corollary 7. The spaces $C_{L^{1}}, L_{C}^{1}$ and their duals are D-P spaces.

4. Remarks and problems.

1. In fact, Theorem 4 is equivalent to the a priori weaker statement that $\bigoplus_{\infty} l^{l}(n)$ is a D-P space. However, the proof of this result does not seem easier and we also use the "Riesz-product technique".

2. Since, by Theorem 4, the space $\bigoplus_{\infty} E_{p}$ has the D-P property, it follows that the $E_{p}$ does not contain uniformly complemented Hilbert spaces of arbitrarily large dimension. This solves a problem raised in $[3$, p. 68].

3. It is unknown if in general the D-P property of $X$ implies the D-P property of $C_{X}$ and $L_{X}^{1}$. Corollary 9 gives us a positive solution to this question in case $X$ is a $C$ or $L^{1}$-space. In fact, we may introduce the sequence $\left(\mathscr{X}_{n}\right)$ of Banach spaces, taking $\mathcal{X}_{1}=C$

$$
\begin{array}{ll}
\mathfrak{X}_{n+1}=C_{\mathscr{X}_{n}} & \text { if } n \text { is even, } \\
\mathfrak{X}_{n+1}=L_{\mathscr{X}_{n}}^{1} & \text { if } n \text { is odd. }
\end{array}
$$

Using similar techniques, it can be shown that all these spaces (and their duals) have D-P property. 
4. So far, we do not know the answer to the following question: Suppose $\left(X_{n}\right)$ a sequence of finite dimensional Banach spaces such that $\bigoplus_{\infty} X_{n}$ is D-P. Does $\bigoplus_{\infty} X_{n}^{*}$ then have the D-P property?

ADDED IN PROOF. Recently, J. Lindenstrauss remarked that the technique explained above yields a lower bound of the order $(\log n)^{1 / 2}$ for the projection onto an $n$-dimensional Hilbert space in $C_{L^{1}}$. This shows that the result in [3] is sharp.

\section{BIBLIOGRAPHY}

1. J. Bourgain, An averaging result for $l^{1}$-sequences and applications to weakly conditionally compact sets in $L_{X}^{1}$, Israel J. Math. 32 (1979).

2. J. Diestel and J. J. Uhl, Vector measures, Math. Surveys, no. 15, Amer. Math. Soc., Providence, R.I., 1977.

3. T. Figiel, J. Lindenstrauss and V. Milman, The dimension of almost spherical sections of convex sets, Acta Math. 139 (1977), 53-94.

4. S. Heinrich, Ultraproducts in Banach space theory, J. Reine Angew. Math. (to appear).

5. J. L. Krivine, Langages à valeurs réelles et applications (to appear).

6. J. Lindenstrauss and H. P. Rosenthal, The $\mathfrak{e}^{p}$-spaces, Israel J. Math. 7 (1969), 325-349.

7. J. Lindenstrauss and L. Tzafriri, Classical Banach spaces, Lecture Notes in Math., vol. 338, Springer-Verlag, Berlin and New York, 1973.

8. Classical Banach spaces. I, Lecture Notes in Math., vol. 92, Springer-Verlag, Berlin and New York, 1977.

9. H. P. Rosenthal, A characterization of Banach spaces containing $l^{\prime}$, Proc. Nat. Acad. Sci. U.S.A. 71 (1974), 2411-2413.

10. The Banach spaces $C(K)$ and $L^{P}(\mu)$, Bull. Amer. Math. Soc. 81 (1975), 763-781.

11. G. Pisier, Une propriété de stabilité de la classe des espaces ne contenant pas $l^{1}, \mathbf{C}$. R. Acad. Sci. Paris Ser. A-B 286 (1978).

12. H. Schaefer, Banach lattices and positive linear operators, Springer-Verlag, Berlin and New York.

13. J. Stern, Some applications of model-theory in Banach space theory (to appear).

Department of Mathematics, Vgue Universiteit Brussel, F7-1050 Brussels, Belgium 